\title{
Efecto del establecimiento del alga invasora Didymosphenia geminata sobre la abundancia de macrocrustáceos en el Río Limay superior, Patagonia, Argentina
}

\author{
Diego A. Añón Suárez ${ }^{1}$ y Ricardo J. Albariño ${ }^{1}$ \\ ${ }^{1}$ GEMA (Grupo de Estudio de Macroinvertebrados Acuáticos), Instituto de Investigaciones en \\ Biodiversidad y Medioambiente (INIBIOMA, UNComahue- CONICET), Quintral 1250, 8400, San Carlos \\ de Bariloche, Río Negro, Argentina \\ E-mail: danon46@gmail.com
}

\begin{abstract}
RESUMEN. Dzidymosphenia geminata (didymo) es una diatomea que desarrolla extensas floraciones que cubren el fondo de ecosistemas lénticos y lóticos determinando fuertes cambios ecológicos. Dada su distribución, abundancia, características tróficas y su importancia como recurso alimentario para vertebrados, los crustáceos Aegla sp. y Samastacus sp. son fundamentales en la transferencia de materia y energía en lagos y ríos patagónicos. En este trabajo se pretende dilucidar si las floraciones algales afectan las poblacionales de ambos crustáceos en el río Limay superior. Durante los veranos de 2016, 2017 y 2018 se muestrearon 4 sitios del río, incluyendo tramos con y sin presencia del alga (CD -con Didymo-y SD -sin Didymo-). La zona litoral de cada sitio fue muestreada en tres hábitats respecto de la línea de costa (costa, somero y profundo). En cada unidad de muestreo se registraron: número de macrocrustáceos, porcentaje de cobertura del alga, desarrollo algal (colonias aisladas, parches), distancia a la costa, profundidad, velocidad de corriente del fondo. Se cuantificaron 855 ejemplares de Aegla riolimayana (10,0 ind / $\mathrm{m}^{2}$ y frecuencia del $73 \%$ ) y sólo 30 de Samastacus spinifrons $\left(0,3\right.$ ind $/ \mathrm{m}^{2}$ y $4 \%$, respectivamente). Dadas estas diferencias, sólo se analizó la distribución de $A$. riolimayana. Su abundancia no difirió entre tramos $C D$ y $S D(P>0,05)$ aunque hubo una tendencia a menores valores en presencia de didymo (mediana $=1 \mathrm{vs.} 2$ ind/cuadrado, respectivamente). El porcentaje de cobertura de didymo fue menor y la abundancia de A. riolimayana fue mayor en el sector de costa respecto al somero y al profundo. Sin embargo la abundancia de esta especie y la cobertura porcentual de didymo no se correlacionaron. Los resultados sugieren que no habría un efecto negativo del alga sobre la distribución de A. riolimayana a escala de tramo.
\end{abstract}

Palabras clave: Especies invasoras, invertebrados bentónicos, litoral, Patagonia Norte.

ABSTRACT. Didymosphenia geminata (didymo) or "rock snot" is a diatom that develops extensive algal blooms covering the bottom of lentic and lotic ecosystems and exerting strong ecological changes. Given its distribution, abundance and trophic characteristics, and the importance as a food resource for aquatic and terrestrial vertebrates, the benthic crustaceans Aeg/a sp. and Samastacus sp. are key components in the transfer of matter and energy in lakes and rivers of Patagonia. We aimed to determine if didymo algal blooms occurring in the littoral zone of the Upper Limay River affect the abundance of both crustaceans. In the summer of 2016, 2017 and 2018 four locations of the river were surveyed, establishing reaches with and without the presence of didymo (CD with Didymo and SD without Didymo, respectively). We sampled the littoral zone in three habitats regarding the distance to shoreline (shore, shallow and deep). At each sample we recorded: number of macrocrustaceans, percentage of algae cover, algal growth characteristics (isolated colonies, mats), distance to the shoreline, depth, current velocity at bottom, among other variables. During the study, 855 Aeg/a riolimayana specimens were quantified with mean abundance of $10.0 \mathrm{ind} / \mathrm{m}^{2}$ and frequency of $73 \%$ and only 30 individuals of Samastacus spinifrons ( $0.3 \mathrm{ind} / \mathrm{m}^{2}$ and $4 \%$, respectively). Given such differences, only $A$. riolimayana was analyzed. Its abundance did not differ between CD and SD reaches $(P>0.05)$, though it was slightly lower in $C D$ reaches (median $=1$ vs. 2 ind./sample, 
respectively). Percent didymo cover was lower and A. riolimayana abundance was higher at shore compared to shallow and deep sectors. However, A. riolimayana abundance and percent didymo cover did not correlate suggesting that at the reach scale there is no negative effect of didymo on the abundance and distribution of $A$. riolimayana.

Keywords: Benthic invertebrates, invader species, littoral, Northern Patagonia.

\section{INTRODUCCIÓN}

Didymosphenia geminata (Lyngbye) Schmidt 1899, es una Bacillariophyta conocida como "didymo" o "moco de roca", característica de cuerpos de agua dulce lénticos y lóticos. Es una de las especies de diatomeas más grandes, con células que alcanzan $100 \mu \mathrm{m}$ de longitud y $35 \mu \mathrm{m}$ de ancho (Kilroy, 2004). Cada célula de $D$. geminata puede producir un largo pedúnculo o tallo por reproducción asexual con el que se fija al sustrato y puede resultar en una mata densa de pedúnculos ramificados distinguibles a simple vista y en ocasiones alcanzando $20 \mathrm{~cm}$ de longitud. En condiciones favorables, sus altas tasas de crecimiento determinan floraciones persistentes en el tiempo que pueden cubrir grandes extensiones del lecho alterando procesos hidrológicos y ecológicos en ríos (Spaulding \& Elwell, 2007). Es sabido que la temperatura, la estabilidad hidrológica y del sustrato, la disponibilidad de luz y el pH son importantes en determinar la capacidad de establecimiento y formación de floraciones ("blooms") a escala local, así como la distribución de la especie a escala regional y global (Kilroy et al., 2008). Varios estudios han descripto el desarrollo de floraciones de $D$. geminata a temperaturas menores a $15^{\circ} \mathrm{C}$, altas intensidades de luz, velocidades de corriente menores a $1 \mathrm{~m} / \mathrm{s}$, así como a pH neutro a moderadamente alcalino (Antoine \& BensonEvans, 1986; Kara \& Sahin, 2001; Kawecka \& Sanecki, 2003; Kilroy et al., 2008; Noga, 2003). En general, los altos valores de biomasa se dan en un amplio rango de velocidad de corriente, con decrecimiento sólo a velocidades mayores de 1 $\mathrm{m} / \mathrm{s}$ (Kilroy et al., 2008), así como en diferentes sustratos estables como lechos rocosos y grandes cantos rodados. Finalmente, se señala a la baja concentración de fósforo (P) disuelto en el agua como factor determinante de las floraciones ya que estimularía el crecimiento en longitud de los pedúnculos de fijación (Stevenson et al., 2008; Kilroy \& Bothwell, 2012; Bothwell et al., 2014).

Respecto a los efectos de sus floraciones sobre el ecosistema acuático, ha sido descripto el significativo aumento en la abundancia de materia orgánica (producto de la proliferación peduncular), de la concentración de clorofila $a$ y de sedimentos atrapados en su matriz (Kilroy et al., 2009; Kilroy \& Bothwell, 2012; Reid \& Torres, 2014). También ha sido demostrada in vitro la capacidad de adsorción de P inorgánico disuelto por parte de matas vivas y muertas, y la alteración de la variación diaria del $\mathrm{pH}$, indicando la importancia que pueden tener las floraciones en alterar ciclos biogeoquímicos a escala de ecosistema (Reid \& Torres, 2014). Varios estudios han descrito los efectos de las floraciones del alga en ríos y arroyos, mayormente en su condición de especie invasora. Cabe recordar que estas floraciones han sido registradas también en áreas donde el alga es nativa (Taylor \& Bothwell, 2014) y no necesariamente ocurren en todos los ríos en donde el alga se encuentra presente.

Por otra parte, se ha registrado que la cobertura del lecho por parte de didymo genera un fuerte cambio en la comunidad de macroinvertebrados. Estos cambios en diferentes regiones del mundo coinciden en provocar un aumento de la densidad total de invertebrados, un aumento de la abundancia de quironómidos y oligoquetos y la reducción en la abundancia de taxa EPT (efemerópteros, plecópteros y tricópteros) mientras el efecto sobre la riqueza taxonómica es despreciable (Gillis \& Chalifour, 2009; Kilroy et al., 2009; Brand y Miserendino, 2014). Más recientemente, Jellyman \& Harding (2016) estudiaron 20 arroyos en la Isla Sur de Nueva Zelandia y además de verificar similares patrones de cambio, demostraron que los 
tramos invadidos con didymo presentaron reducción en la tasa de deriva de invertebrados EPT (recurso trófico fuertemente explotado por peces) y una drástica reducción en la biomasa de los peces, especialmente la trucha marrón (Salmo trutta Linnaeus, 1758). Es interesante notar que a la fecha no se han publicado estudios que analicen los efectos de la invasión de didymo sobre macrocrustáceos, quienes representan un componente clave en la trama trófica de ríos y arroyos.

En Sudamérica, las primeras floraciones documentadas ocurrieron en la cuenca del río Futaleufú, cuenca binacional Chileno-Argentina (Patagonia). El primer registro data de abril de 2010 en el río Espolón, tributario del río Futaleufú (Reid \& Torres, 2014). En septiembre de 2010 fue reportada por primera vez en el río Futaleufú en Argentina (Sastre et al., 2013). A pesar de las medidas efectuadas por diferentes instituciones nacionales y provinciales para minimizar su dispersión (A.P.N. 2015), el alga ha invadido una serie de ambientes fluviales y lacustres extendiéndose a lo largo de la Patagonia Andina desde Neuquén a Tierra del Fuego. Como parte de la comunidad de invertebrados, en ríos y lagos de Patagonia se encuentran presentes dos macrocrustáceos (Crustacea, Decapoda): la langosta Samastacus spinfrons (Philippi, 1882) y la "pancora" Aegla riolimayana (Schmitt, 1942) (Lopretto, 1995). Estos decápodos son endémicos de Sudamérica (Lopretto, 1995; Morrone \& Lopretto, 1994) y presentan relaciones zoogeográficas gondwánicas, distribuyéndose en el sur del continente, a ambos lados de la Cordillera de los Andes (Chile y Argentina). En ríos y lagos del Parque Nacional Nahuel Huapi (P.N.N.H.), ambos macrocrustáceos constituyen un ítem alimentario importante para peces autóctonos, Percichthys trucha (Valenciennes, 1833), e introducidos - Oncorhynchus mykiss (Walbaum, 1792) y S. trutta - (Macchi et al., 1999). También son fuente de alimento de anfibios (Fransozo et al., 2003), aves y mamíferos como el huillín o lobito de río, Lontra provocax (Thomas, 1908), emblema del P.N.N.H., del cual constituyen el 99 \% de la dieta (Chehebar, 1985; MedinaVogel \& González-Lagos, 2008; Rudolph, 2002). Dada su distribución, abundancia y características tróficas, y su importancia como recurso alimentario, ambos macrocrustáceos constituyen elementos claves en las tramas tróficas de lagos y ríos, cumpliendo un rol importante en la transferencia de energía (Aued, 2003; Chehebar, 1985; Fransozo et al., 2003; Jara et al., 2006; Rudolph, 2002).

Como se mencionó anteriormente, a partir del registro de las primeras floraciones en la Patagonia chilena y argentina en el 2010 , se evidenció una rápida expansión del alga, registrándose en el área del P.N.N.H en 2011 (río Manso inferior), y en 2012 en el lago Nahuel Huapi y en el río Limay. Las floraciones de $D$. geminata han generado gran preocupación debido a los posibles impactos negativos socio-ecológicos, principalmente cuando éstas ocurren en ríos de aguas cristalinas, donde la pesca deportiva de salmónidos constituye un importante recurso socio-económico para el área (Taylor \& Bothwell, 2014). A la fecha se desconocen los efectos que tiene didymo sobre la comunidad de macroinvertebrados en esta zona, incluyendo a las poblaciones de macrocrustáceos. Dada la relevancia trófica de estos macrocrustáceos para especies nativas de la región Patagónica como el huillín cuyo estatus de conservación es en peligro (Sepúlveda et al., 2015), este conocimiento representa información vital para la Administración de Parques Nacionales (Argentina). Por otra parte, la biología y ecología de ambos macrocrustáceos ha sido poco estudiada, especialmente en la Patagonia Argentina, lo que representa un aporte a la conservación de la biodiversidad regional. Por ello, el objetivo principal de este estudio fue evaluar el efecto del establecimiento del alga $D$. geminata en la abundancia y distribución de $A$. riolimayana y $S$. spinifrons en el río Limay superior.

\section{MATERIALES Y MÉTODOS \\ Área de estudio}

El Río Limay es uno de los grandes sistemas fluviales de la Patagonia Norte, nace del drenado del lago Nahuel Huapi $\left(41^{\circ} 3^{\prime} 18,52^{\prime \prime} \mathrm{S}, 71^{\circ} 9^{\prime}\right.$

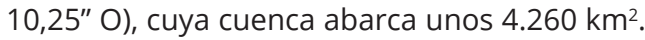
La misma está caracterizada mayormente por la presencia de bosque templado dominado 
por Nothofagus spp. y el ciprés de la cordillera (Austrocedrus chilensis (D.Don) Pic-Serm. \& Bizzarri), junto a un sotobosque caracterizado por diferentes especies arbustivas y herbáceas. El río Limay es alimentado por precipitaciones pluviales y níveas, presentando un ciclo anual bimodal con un pico de descarga en invierno por lluvias y otro de mayor caudal en primavera debido al deshielo. El caudal medio anual es de $224 \mathrm{~m}^{3} / \mathrm{s}$, y las características químicas del agua están mayormente influenciadas por el lago Nahuel Huapi, con un $\mathrm{pH}$ cercano al neutro $(7,5)$, baja conductividad $(39,3 \mu \mathrm{S} / \mathrm{cm})$ y baja concentración de nutrientes (fósforo reactivo soluble $P R S=1,9 \mu \mathrm{g} / \mathrm{L}$ y nitrógeno inorgánico disuelto NID=9,8 $\mu \mathrm{g} / \mathrm{L}$ ) (Díaz et al., 2007). El sustrato del lecho es grueso, conformado por bloques, guijones (canto rodado), guijarros y parches de grava y arena. Su cauce presenta extensiones dominadas mayormente por un canal único y extensiones donde hay canales principales y secundarios. Si bien toda la extensión del río presenta un importante valor cultural y turístico, existen sitios de gran valor escénico. En el río se desarrolla una importante actividad de pesca deportiva en base a los salmónidos introducidos, con pesca desde las márgenes o embarcaciones sin motor. Además se desarrollan actividades recreativas de descenso en balsas y canotaje. En las últimas dos décadas, el paraje Villa Llanquín presenta un creciente desarrollo urbanístico.

El presente estudio se realizó en un segmento de aproximadamente $17 \mathrm{~km}$, comprendido entre los parajes denominados Estancia Fortín Chacabuco y Villa Llanquín, localizado a $40 \mathrm{~km}$ de Bariloche y $25 \mathrm{~km}$ de la embocadura del río. Este segmento de río no recibe tributarios fluviales de gran magnitud, lo que determina una mayor homogeneidad del cauce y de las variables bióticas y abióticas que interactúan en el ecosistema.

\section{Diseño de muestreo}

Se seleccionaron sitios con presencia visible del alga cubriendo el lecho fluvial y sitios sin presencia macroscópica. La presencia visible del alga se estableció como la identificación de colonias individualizables sobre sustrato inorgánico, denominadas coloquialmente "botones", originados por la fijación de uno o más individuos al sustrato, que al crecer y dividirse asexualmente desarrolla pedúnculos característicos que se multiplican de manera dendrítica (Spaulding \& Elwell, 2007). Se establecieron 4 sitios de muestreo, cada uno contemplando un tramo sin presencia del alga didymo denominado SD (sin didymo), donde la superficie cubierta por el alga fuera mínima (menor al 15\%) y un tramo contiguo con establecimiento extendido del alga llamado CD (con didymo), es decir cuando la superficie cubierta por colonias y matas algales fuera alta (mayor al 50\%). Los sitios de muestreo fueron establecidos en sentido aguas abajo y se denominaron: Rincón Chico, Fortín Chacabuco, La Perca y Villa Llanquín. Este último, el más alejado del Lago Nahuel Huapi, evidenció una presencia incipiente de didymo (limitada a pequeñas colonias aisladas solo en unas pocas piedras del litoral) por lo que se consideró al sitio como un tramo control (SD). Los muestreos se realizaron durante febrero-marzo, en tres años consecutivos: 2016, 2017 y 2018. Durante este período, el caudal del río se encuentra en disminución gradual luego de su máximo asociado al deshielo de las nacientes y a la fuerte reducción de las precipitaciones estacionales, determinando menores velocidades de corriente lo que facilita el muestreo. Una vez definidos los tramos CD y SD en cada sitio, se realizó un muestreo estratificado en la zona litoral, considerando tres hábitats definidos por la distancia a la orilla: costa (0-1 m), somero (3-5 m) y profundo (7-9 $\mathrm{m}$ ). Debido a que los muestreos se realizaron mediante vadeo, las muestras en el hábitat profundo no se extendieron más allá de los $70 \mathrm{~cm}$ de profundidad (aproximadamente $10 \mathrm{~m}$ de la costa). El muestreo consistió en colocar sobre el lecho un cuadrado metálico de $50 \times 50 \mathrm{~cm}$ (unidad muestral) a fin de medir sus variables ambientales y cuantificar la presencia y abundancia de los macrocrustáceos. Los cuadrados fueron colocados al azar sobre el lecho y distanciados entre sí dos metros o más a fin de asumir independencia de las muestras. El muestreo siempre se realizó en sentido aguas arriba. En cada tramo (cuatro tramos SD y tres tramos $(D)$ se tomaron seis muestras por hábitat (costa, somero y profundo). En cada muestra se determinó: porcentaje de cobertura 
del alga mediante inspección visual, distancia a la costa, profundidad, velocidad media de la corriente del fondo y tamaño del sustrato. Para esto se usó una cinta métrica, una vara graduada y un flujómetro Global Water F101 (Xylem Inc., EEUU). La velocidad media del fondo fue medida colocando el flujómetro apoyado sobre el sustrato en la parte central de cada unidad de muestreo y registrando su velocidad media durante aproximadamente 15 segundos. El sustrato fue clasificado como bloque (mayor o igual a $25 \mathrm{~cm}$ ), canto rodado ( 8 a $25 \mathrm{~cm}$ ), guijarro $(4$ a $8 \mathrm{~cm})$ y arena + grava $(<4 \mathrm{~cm})$ y se registró visualmente su cobertura y "empotramiento", es decir el grado de sedimentación de bloques, cantos rodados y guijarros en la matriz del lecho con una escala de 0 a 2 (totalmente suelto, parcialmente suelto y totalmente empotrado en sedimento fino). Luego de registrar las variables ambientales, se procedió a contar los macrocrustáceos del fondo usando visores subacuáticos y levantando cuidadosa y gradualmente los sustratos dentro del cuadrado de forma de registrar los que no estuvieran expuestos. Esta operación fue realizada por dos personas, mientras una tercera se dispuso aguas abajo del cuadrado con una red de pateo (malla $2 \mathrm{~mm}$ ) a fin de colectar a los individuos que derivaran desde el fondo hacia la columna de agua, especialmente en las muestras con alta velocidad de corriente. En general, los individuos siempre permanecieron en el fondo o bien quietos o reptando lentamente lo que permitió una cuantificación certera de su abundancia. Finalmente, en cada hábitat dentro de cada tramo de muestreo se midieron las siguientes variables ambientales: temperatura del agua, conductividad, concentración de oxígeno disuelto, profundidad y ancho húmedo del cauce. En cada sitio se colectó una muestra de agua que fue filtrada en el campo y refrigerada en oscuridad hasta su análisis químico en laboratorio a fin de cuantificar PRS (por reducción del complejo fosfomolíbdico con ácido ascórbico), $\mathrm{NO}_{3}^{-}+\mathrm{NO}_{2}^{-}$(por reducción en columna de cadmio y diazotización) y $\mathrm{NH}_{4}{ }^{+}$ (por método del azul-indofenol) y lectura en espectrofotómetro (APHA, 1992). Los resultados de las fracciones de nitrógeno inorgánico disuelto son reportados como la suma de sus concentraciones $\left(\mathrm{NO}_{3}^{-}+\mathrm{NO}_{2}^{-}+\mathrm{NH}_{4}^{+}\right)$.
Durante todo el estudio se siguió el protocolo de desinfección de equipo y ropa de trabajo recomendado por la Intendencia del Parque Nacional Nahuel Huapi (que busca evitar la dispersión de propágulos del alga invasora) usando solución salina al 5\%, preparada con sal de mesa, para sumergir materiales y equipos al menos durante 5 minutos.

\section{Análisis de datos}

La toma de muestras en tres años consecutivos tuvo por objeto aumentar la potencia del estudio incrementando el número de muestras y la ventana de tiempo analizada, por lo que el análisis de datos se realizó agrupando las tres campañas. Aunque reconocemos que podría existir una variación interanual, la consideramos como un forzante no relevante en términos de la causalidad que procuramos poner a prueba. Dada la heterogeneidad de los datos que determinó su falta de normalidad aún luego de procurar transformarlos, el análisis de diferencias de las variables abióticas (distancia a la costa, profundidad del fondo y velocidad media en el fondo) y las variables respuesta (cobertura porcentual de didymo y abundancia de Aeg/a) entre tramos o entre hábitats fue realizado con la prueba no paramétrica de varianzas de Kruskal-Wallis seguida por pruebas pos hoc de Dunn (Quinn \& Keough, 2002). Además se exploraron las relaciones entre las variables respuesta mencionadas y la velocidad media en el fondo (variable crítica de las condiciones fluviales sensu Gordon et al. (2004) y la relación entre la cobertura porcentual de didymo y la abundancia de Aegla usando modelos de regresión. En estos modelos se probó la significancia de las relaciones sin considerar el hábitat o el tramo con el fin de analizar la existencia de patrones de distribución independizados de la categorización establecida a priori (tramos CD y SD). Para esto se exploró el ajuste de las variables a dos modelos de regresión, uno lineal simple $\left(y=a+x^{*} b\right)$ y otro exponencial negativo $\left.\left(y=a^{*} e^{\left(-b^{\star} \times\right.}\right)\right)$ usando el procedimiento de ajuste de curvas del programa SigmaPlot 11.0 (Systat Software Inc.). Debido a que en todos los casos los datos, aún luego de transformados no cumplieron los supuestos de normalidad, el modelo de regresión lineal 
Tabla 1. Media aritmética de la profundidad, distancia a la costa y velocidad del fondo de todas las unidades de muestreo en los tres hábitats muestreados en tramos con y sin didymo (CD y SD, respectivamente).

Table 1. Mean values of depth, shoreline distance and bottom current velocity for all samples at three sampled habitats in reaches: with and without didymo (CD and SD, respectively).

\begin{tabular}{|c|c|c|c|c|c|c|c|c|c|}
\hline & \multicolumn{3}{|c|}{ Totales } & \multicolumn{3}{|c|}{$C D$} & \multicolumn{3}{|c|}{ SD } \\
\hline & Costa & Somero & Profundo & Costa & Somero & Profundo & Costa & Somero & Profundo \\
\hline $\begin{array}{c}\text { Distancia a la } \\
\text { Costa }(\mathrm{cm})\end{array}$ & 47 & 397 & 826 & 50 & 407 & 889 & 45 & 389 & 768 \\
\hline $\begin{array}{l}\text { Profundidad } \\
\text { Media }(\mathrm{cm})\end{array}$ & 5 & 26 & 39 & 6 & 23 & 39 & 5 & 28 & 40 \\
\hline $\begin{array}{l}\text { Velocidad } \\
\text { corriente } \\
\text { Fondo }(\mathrm{cm} / \mathrm{s})\end{array}$ & $<3$ & 16 & 18 & $<3$ & 12 & 15 & $<3$ & 18 & 21 \\
\hline
\end{tabular}

fue desechado y alternativamente se analizó la correlación no paramétrica en rangos de Spearman. Todas las pruebas fueron analizadas considerando un valor alfa de 0,05 con el programa SigmaPlot 11.0.

\section{RESULTADOS}

Las características físicas del agua en los tres años de muestreo presentaron condiciones similares entre los tramos CD y SD (conductividad: 36,4 vs. $35,2 \mu \mathrm{S} / \mathrm{cm}$; temperatura del agua: 17,7 vs. $18,7^{\circ} \mathrm{C}$; concentración de oxígeno disuelto: 8,7 vs. $8,9 \mathrm{mg} / \mathrm{L}$, respectivamente).

Las muestras tomadas en los tres hábitats litorales seleccionados (Costa-SomeroProfundo) en el período 2016-2018, mostraron un aumento en la velocidad del agua conforme aumentaba la profundidad (Tabla 1). La profundidad media y la distancia media a la costa no presentaron mayores diferencias entre los tramos invadidos con didymo y los tramos sin el alga $(n=343$, Kruskal-Wallis para profundidad media: $\mathrm{H}_{1,342}=0,625, \mathrm{P}=0,429$ y para distancia a la costa: $\left.\mathrm{H}_{1,342}=2,058, \mathrm{P}=0,151\right)$. Por otra parte, la velocidad del agua a nivel del sustrato (velocidad en el fondo) fue un $47 \%$ mayor en los tramos sin didymo (Kruskal-Wallis $\mathrm{H}_{1,186}=18,725, \mathrm{P}<0,001$ ). Estas diferencias corresponden a los hábitats somero y profundo, ya que en el hábitat Costa la velocidad del agua fue despreciable en ambos tramos ( $<3 \mathrm{~cm} / \mathrm{s})$ (Tabla 1).

Las concentraciones de nutrientes medidas en el litoral del río fueron similares entre sitios y muy bajas, con valores promedio de 5,7 $\pm 2,0$ (NID) y 2,2 \pm 0,4 (PRS) (media \pm e.s.) condición que caracteriza las aguas del río y las del lago que lo abastece.

Del total de cuadrados relevados durante el estudio (343), se contaron 855 individuos de Aeg/a riolimayana y 30 individuos de Samastacus spinifrons (Tabla 2), con una relación de 28:1. En promedio la abundancia de S. spinifrons en el litoral del río fue 0,3 ind/cuadrado mientras la de $A$. riolimayana alcanzó una media de 10,0 ind/cuadrado. Similarmente las frecuencias de ocurrencia en las muestras fueron de $4 \%$ y $73 \%$, respectivamente. La escasa abundancia de $S$. spinifrons y su baja frecuencia de ocurrencia (Tabla 2) determinaron que el análisis de datos se realice exclusivamente para $A$. riolimayana. En particular, la abundancia media y la frecuencia de ocurrencia de $A$. riolimayana en los cuadrados fueron levemente superiores en los tramos $C D$ con respecto a los de SD (Tabla 2 ).

Los tres hábitats litorales estudiados mostraron patrones contrastantes de distribución de $D$. geminata y A. riolimayana. La cobertura algal difirió entre los hábitats estudiados, siendo mayor en los hábitats somero y profundo respecto al de costa (Figura 1.a, Kruskal-Wallis $\mathrm{H}_{2,341}=15,611, \mathrm{P}<0,001$, prueba pos hoc Dunn $\mathrm{P}<0,05)$. Por el contrario, la abundancia de $A$. riolimayana fue mayor en costa respecto a la de somero y profundo (Figura 1.b, Kruskal-Wallis $H_{2,341}=13,759, P<0,001$, prueba pos hoc Dunn $\mathrm{P}<0,05$ ).

La cobertura de didymo fue mayor en los tramos $C D$ (Figura 2.a, Kruskal-Wallis $H_{1,342}=58,909$, $\mathrm{P}<0,001)$. Por su parte, la abundancia y la frecuencia de aparición de A. riolimayana en los cuadrados presentaron una tendencia a 
Tabla 2. Abundancia de Aegla riolimayana y Samastacus spinifrons en la zona littoral del Río Limay superior y abundancia de $A$. riolimayana en tramos con y sin didymo (CD y SD). Los datos corresponden a los tres años muestreados. La abundancia porcentual es la abundancia relativa de ambas especies y la frecuencia porcentual es el número de muestras con presencia de cada especie.

Table 2. Abundance of Aegla riolimayana and Samastacus spinifrons in the litoral zone of upper Limay River and abundance of $A$. riolimayana at reaches with (CD) and without (SD) didymo. Data refer to for the three sampling years. Percent abundance is the relative abundance of both species and percent frequency means the number of samples with presence of each species.

\section{Totales}

Aegla riolimayana

\begin{tabular}{|c|c|c|c|c|}
\hline & Samastacus spinifrons & Aegla riolimayana & $C D$ & SD \\
\hline Nro. de cuadrados totales & \multicolumn{2}{|c|}{343} & 154 & 189 \\
\hline Nro. de individuos contados & 30 & 855 & 348 & 507 \\
\hline Abundancia \% & 3,4 & 96,6 & & \\
\hline Nro. individuos / cuadrado & 0,1 & 2,5 & 2,3 & 2,7 \\
\hline Nro. individuos $/ \mathrm{m}^{2}$ & 0,3 & 10,0 & 9,7 & 10,7 \\
\hline Frecuencia \% & 4,1 & 72,9 & 70,1 & 75,1 \\
\hline
\end{tabular}

ser mayores en los tramos CD (Tabla1). Sin embargo, la abundancia expresada por su mediana no fue diferente entre tramos (Figura 2.b, Kruskal-Wallis $\left.\mathrm{H}_{1,342}=2,026, \mathrm{P}=0,155\right)$.

En los tramos clasificados como SD, el alga didymo también estuvo presente, aunque en muy baja abundancia (Figura 2.a). El porcentaje de cobertura no superó el 25\% y se caracterizó por la presencia de pequeñas colonias aisladas creciendo sobre el sustrato $(1-3 \mathrm{~cm}$ de diámetro $y<3 \mathrm{~cm}$ de espesor). En los tramos CD, la cobertura de didymo alcanzó valores del 90\%. En algunos sectores el alga se presentó formando colonias individuales ocupando un mismo sustrato en forma variable, mientras en otros se extendió cubriendo varios sustratos formando una mata densa y espesa $(20-40 \mathrm{~cm}$ de diámetro y 2-4 cm de espesor).

La cobertura de didymo y la velocidad en el fondo no se correlacionaron linealmente (rho de Spearman=-0,006, $\mathrm{P}=0,938, \mathrm{n}=193$ ), pero sí lo hicieron siguiendo una función exponencial

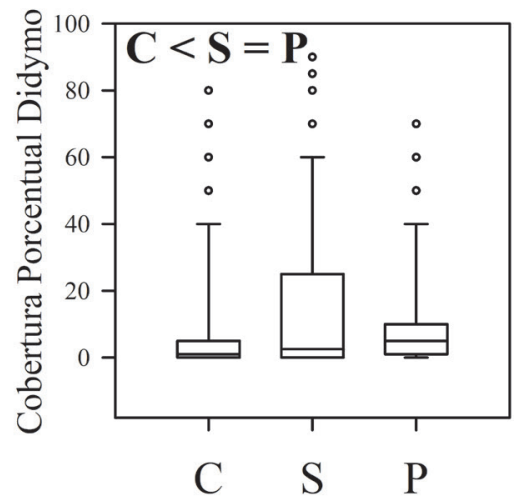

b

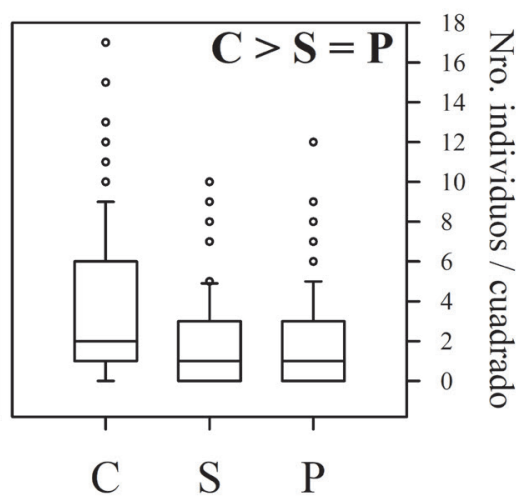

Figura 1.a. Cobertura porcentual de didymo; b. número de individuos de Aegla riolimayana por unidad muestral (cuadrado de $50 \times 50 \mathrm{~cm}$ ) con relación a la distancia a la orilla: C (costa), S (sector somero), P (sector profundo). Los valores de la caja representan la mediana y sus cuartiles 1 y 3 y las barras de error los deciles 1 y 9 . Se indica la comparación significativa entre hábitats, (prueba de Kruskal-Wallis y prueba a posteriori de Dunn, $\mathrm{P}<0,05$ ).

Figure 1.a. Percent cover of didymo; b. number of individuals of Aegla riolimayana per sampling unit ( 50 × $50 \mathrm{~cm}$ metal frame) at three distances from river shoreline: C (shore sector), S (shallow sector), P (profundal sector). Values within the box represent median and quartiles 1 and 3, and error bars are deciles 1 and 9. Statistical differences among habitats are shown, (Kruskal-Wallis test, and post hoc Dunn's test, $\mathrm{P}<0.05$ ). 
a

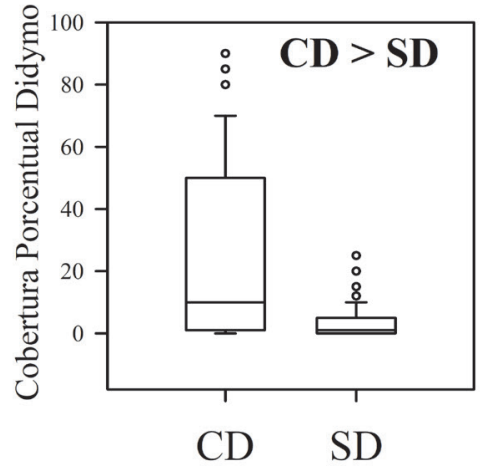

b

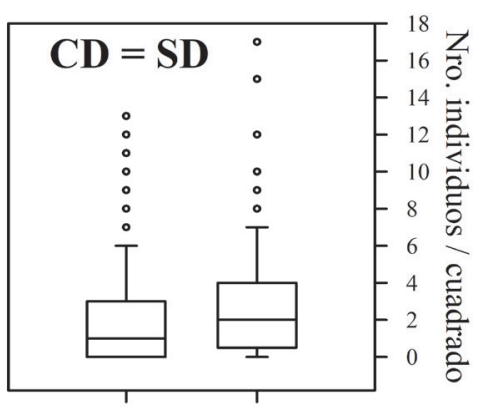

Figura 2.a. Cobertura porcentual de didymo; b. número de individuos de Aeg/a riolimayana por unidad muestral $(50 \times 50 \mathrm{~cm})$, considerando tramos con y sin presencia del alga Didymosphenia geminata (CD vs SD). Se indica la comparación significativa entre tramos (prueba de Kruskal-Wallis, $\mathrm{P}<0,05$ ).

Figure 2.a. Percent cover of didymo; b. number of individuals of Aeg/a riolimayana per sampling unit ( $50 \times 50$ $\mathrm{cm}$ ) at reaches with (CD) and without (SD) presence of Didymosphenia geminata. Statistical differences between reaches are shown (Kruskal-Wallis test, $\mathrm{P}<0.05)$.

negativa (Figura 3.a, Cobertura Porcentual didymo $=39,82$ * e $(-0,12$ * Velocidad Fondo $), \mathrm{R}^{2}$ aj. $=0,25$, Modelo Regresión No Lineal: $\mathrm{F}_{1,193}=65,47$, $\mathrm{P}<0,001)$. En contraste, la abundancia de $A$. riolimayana no se relacionó con la velocidad en el fondo (Figura 3.b) ni de manera lineal (rho de Spearman=-0,040, $\mathrm{P}=0,583, \mathrm{n}=193$ ) $\mathrm{ni}$ con un decaimiento exponencial $\left(F_{1,341}=1,966, P=0,162\right)$.
Cuando se analizó la relación entre las abundancias de didymo y de $A$. riolimayana, ambas variables no ajustaron significativamente al modelo de regresión exponencial negativa $\left(F_{1,341}=1,966, P=0,162\right)$ y tampoco se correlacionaron linealmente (rho de Spearman=-0,091, $P=0,093, n=343$ ). Cabe resaltar que, si bien los mayores valores de

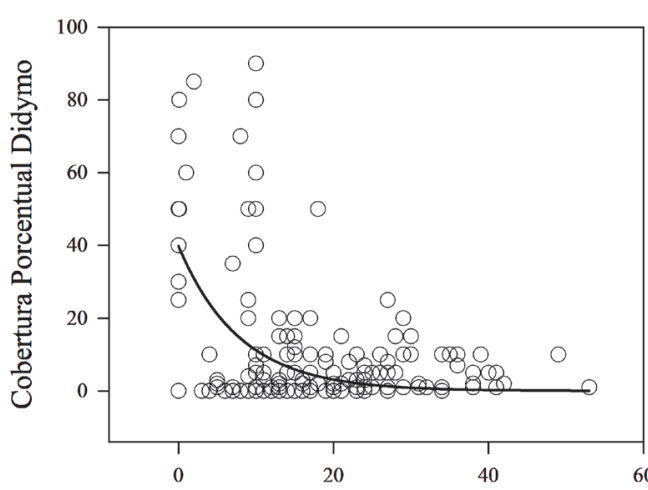

b

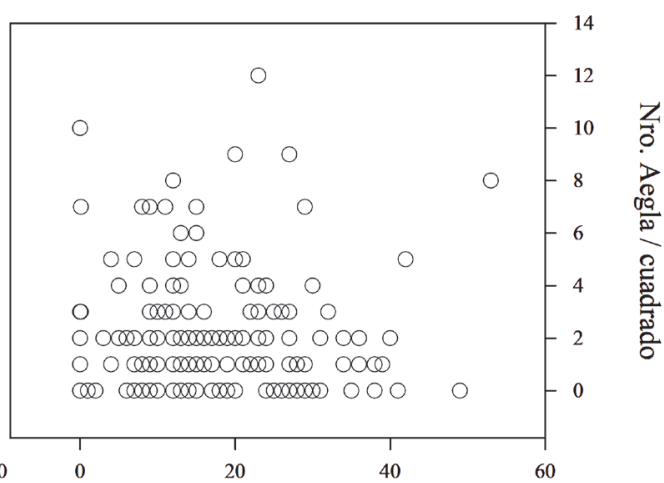

Velocidad del Fondo $(\mathrm{cm} / \mathrm{s})$

Figura 3.a. Cobertura porcentual de didymo; b. número de individuos de Aegla riolimayana por unidad muestral $(50 \times 50 \mathrm{~cm}$ ) en relación con la velocidad de agua del fondo. La línea sólida muestra el modelo de regresión no lineal ajustado entre \% Cobertura de didymo y la velocidad de corriente en el fondo: Cobertura Porcentual didymo $=39,821$ * $\mathrm{e}(-0,127$ * Velocidad Fondo $), \mathrm{R}^{2} \mathrm{aj} .=0,25, \mathrm{~F}_{1,193}=65,47, \mathrm{P}<0,0001$.

Figure 3.a. Percent cover of didymo; b. number of individuals of Aeg/a riolimayana per sampling unit ( $50 \times 50$ $\mathrm{cm}$ ) related to bottom current velocity. Solid line shows a non-lineal regression model for $\%$ didymo cover and bottom current velocity: $\%$ didymo Cover $=39.821$ * $\left.\mathrm{e}^{-0,127 \text { * bottom velocity }}\right), \mathrm{R}^{2} \mathrm{adj} .=0.25, \mathrm{~F}_{1,193}=65.47, \mathrm{P}<0.0001$. 


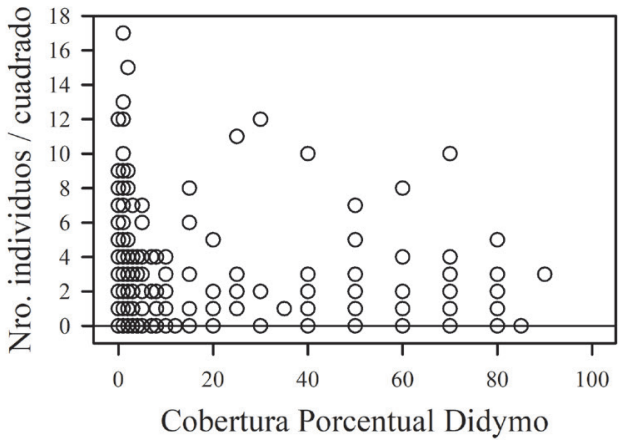

Figura 4. Relación entre el número de individuos de Aegla riolimayana por unidad muestral (cuadrado de 50 x $50 \mathrm{~cm}$ ) y la cobertura porcentual de didymo.

Figure 4. Relationship between the number of individuals of Aeg/a riolimayana per sampling unit (50 $x 50 \mathrm{~cm}$ ) and the percent cover of didymo.

cobertura algal estuvieron asociados a valores bajos o nulos de abundancia de la A. riolimayana (Figura 4), a bajos valores de cobertura algal también se registraron valores bajos y nulos en la abundancia de esta especie.

\section{DISCUSIÓN}

Nuestro estudio revela que el establecimiento de floraciones algales de $D$. geminata no tiene efectos aparentes sobre la distribución de $A$. riolimayana en la zona litoral del río Limay. Esto sugiere que son otros los factores ambientales que estarían afectando su presencia y abundancia en esa zona del río. El estudio también mostró que la presencia de $S$. spinifrons es escasa o nula en las zonas litorales de corredera y pozón, por lo que se encontraría en zonas más profundas. Este patrón se presenta también en el lago Nahuel Huapi, donde A. riolimayana se localiza preferentemente en el litoral rocoso mientras S. spinifrons lo hace en las zonas más profundas y de fondo limo/arenoso (Lucci, 2015). Ese estudio muestra que S. spinifrons se encuentra habitando el fondo preferentemente a partir de los $10 \mathrm{~m}$ de profundidad, habitando lechos mayormente limosos, mientras que A. riolimayana se distribuye hasta $10 \mathrm{~m}$ en la zona litoral en fondos mayormente rocosos. Asimismo, tanto en ríos como en lagos chilenos,
Rudolph (2002) ha observado un patrón de distribución similar para Samastacus. Considerando que las floraciones del alga didymo se observaron exclusivamente en la zona litoral, es posible suponer que los efectos sobre la población de Samastacus sean despreciables.

La presencia del alga en la zona litoral del Río Limay presentó cierto grado de estratificación, siendo más abundante en su parte más profunda (sitios denominados Somero y Profundo, entre los 20 y $70 \mathrm{~cm}$ de profundidad aproximadamente) y menor en la zona cercana a la orilla (Costa $<10 \mathrm{~cm}$ de profundidad). A pesar de que el muestreo del sustrato fue restringido a la zona litoral del río, el registro ocular nos permitió determinar la desaparición de matas y la reducción de colonias en zonas lindantes más profundas. Este patrón es consistente con los modelos desarrollados para explicar la dinámica espacial y temporal de la floración del alga didymo (Bray et al., 2016, 2017; Cullis et al. 2012). Estos modelos indican que en condiciones adecuadas de luz, temperatura y nutrientes (fundamentalmente bajas concentraciones de P inorgánico disuelto) didymo puede desarrollar floraciones agresivas (Cullis et al., 2012, Kilroy \& Bothwell 2012; Montecino et al., 2014; Stevenson et al., 2008, Sundareshwar et al., 2011). Por el contrario, la baja estabilidad del lecho asociada a tamaños de partícula de sustrato pequeños, a su mínimo empotramiento en la matriz del lecho y a una alta velocidad de corriente, son factores que reducen la capacidad de colonizar el fondo y desarrollar floraciones (Cullis et al. 2012, Jellyman \& Harding 2016). En el área muestreada, el lecho del río se encuentra dominado por cantos rodados y mayormente todo el sustrato contenido en las unidades de muestreo se encontró suelto sobre el fondo (es decir, con escaso grado de sedimentación), sugiriendo una muy buena calidad de hábitat para la comunidad de invertebrados bentónicos, incluyendo a los macrocrustáceos. El bajo grado de sedimentación determina que los sustratos sean más propensos a moverse cuando la velocidad de la corriente es alta (fenómeno conocido como acarreo de fondo (Gordon et al., 2004). Como la velocidad de corriente 
en el fondo aumentó con la profundidad, es probable que esta variable junto con la disminución de la intensidad de luz, sean determinantes a la hora de limitar la expansión de las floraciones en la zona más profunda del río, como lo sugieren Cullis et al. (2012). Esta observación visual es consistente con los datos de velocidad de corriente obtenida en los cuadrados muestreados, donde la cobertura algal de didymo decayó exponencialmente con el aumento de la velocidad de corriente en el fondo.

Por su parte, $A$. riolimayana también mostró una distribución diferencial en el litoral del río aunque en oposición al patrón observado en didymo; su abundancia disminuyó a mayor distancia de la costa (mayor profundidad). Sin embargo, su abundancia no tuvo relación con la velocidad medida en el fondo, ni con la abundancia de didymo. El único estudio que analizó la distribución de especies de Aegla en relación con la velocidad de corriente, entre otras 10 variables ambientales corresponde a Bücker et al. (2008) en un arroyo del sur de Brasil, y no encontró que esta variable explicara las abundancias y distribuciones de Aeg/a itacolomiensis (Bond-Buckup \& Buckup, 1994) o Aegla platensis (Schmitt, 1942). En otros ríos de la zona pero de menor tamaño y cuya profundidad permite vadearlos, A. riolimayana, es el único macrocrustáceo presente y muestra un patrón parecido al del río Limay, donde su mayor abundancia ha sido observada cerca de la costa (Arroyo Ñireco y Arroyo Ñirihuau, Albariño obs.pers.). En contraste, en ríos pequeños de Patagonia norte en Chile (10 $\mathrm{m}$ de ancho y hasta $1 \mathrm{~m}$ de profundidad) Parra et al. (2011) demostraron que Aegla araucaniensis (Jara, 1980) puede ocupar todo el lecho cuando se encuentra sola o bien restringir su distribución a la costa cuando cohabita con otras especies competidoras como Aegla abtao (Schmitt, 1942) y Aegla denticulata (Nicolet, 1842). Por lo tanto es posible que las mayores abundancias de $A$. riolimayana sean exclusivas de la zona litoral particularmente cercanas a la costa representando una condición específica de uso del hábitat.

Nuestro estudio representa el primer análisis del efecto del establecimiento del alga invasora D. geminata sobre la abundancia y distribución de poblaciones de macrocrustáceos de agua dulce. Los resultados muestran que la abundancia y distribución de $A$. riolimayana fue independiente de la registrada para el alga didymo. Nuestra evidencia indica entonces que estos macrocrustáceos no están siendo afectados negativamente por la invasión de didymo en la escala espacial abordada (escala de tramo).

\section{AGRADECIMIENTOS}

Agradecemos a la Intendencia del Parque Nacional Nahuel Huapi y especialmente a las Lic. Susana Seijas, Gloria Fernández Cánepa y Carla Pozzi por la colaboración y financiación brindada en el marco de un convenio entre la Intendencia del Parque y el Centro Regional Universitario Bariloche. También a los encargados de las propiedades Fortín Chacabuco (Nicolás Rodríguez) y La Perca (Lalo Martínez) por permitirnos el acceso a los sitios de muestreo. Por último queremos agradecer a los técnicos del INIBIOMA Pablo Alvear y Ariel Mayoral por su asistencia en el trabajo de campo.

\section{BIBLIOGRAFÍA}

APHA (1992). Standard Methods for the Examination of Water and Wastewater. 18th Edition. Washington, DC: American Public Health Association (APHA), American Water Works Association (AWWA) and Water Pollution Control Federation (WPCF).

Administración de Parques Nacionales (A.P.N). Parque Nacional Nahuel Huapi (2015). Fernández Cánepa, G. Informe: Monitoreo de la presencia de Didymosphenia geminata en el PNNH, primavera 2014-verano 2015. CUDAP: TRI-PNA: 28347/2015.

Antoine, S. E. \& Benson-Evans, K. (1986). Spatial and temporal distribution of some interesting diatom species in the Wye River system, Wales, UK. Limnologica, 17: 79-86.

Aued, M. B. (2003). Relevamiento de Decápodos en el PN Nahuel Huapi. Distribución, Abundancia e 
implicancias ecológicas. Informe Parques: Beca Formación Secretaría de Ciencia y Técnica de la Nación- Fundación Profauna.

Bothwell, M. L., Taylor, B. W. \& Kilroy, C. (2014). The Didymo story: the role of low dissolved phosphorus in the formation of Didymosphenia geminata blooms. Diatom Research, 29(3): 229236.

Brand, C. y Miserendino, M. L. (2014). Evaluación de los efectos de la invasión de Didymosphenia geminata en la comunidad del bentos del Río Futaleufú (Patagonia, Argentina). Biología Acuática, 29: 110.

Bray, J., Harding, J. S., Kilroy, C., Broady, P. \& Gerbeaux, P. (2016). Physicochemical predictors of the invasive diatom Didymosphenia geminata at multiple spatial scales in New Zealand rivers. Aquatic Ecology, 50(1): 1-14.

Bray, J., Kilroy, C., Gerbeaux, P. \& Harding, J. S. (2017). Ecological eustress? Nutrient supply, bloom stimulation and competition determine dominance of the diatom Didymosphenia geminata. Freshwater Biology, 62(8): 1433-1442.

Bücker, F., Gonçalves, R., Bond-Buckup, G. \& Melo, A. S. (2008). Effect of Environmental Variables on the Distribution of two Freshwater Crabs (Anomura: Aeglidae). Journal of Crustacean Biology, 28: 248-251.

Chehebar, C. (1985). A survey of the southern river otter Lutra provocax Thomas in Nahuel Huapi National Park, Argentina. Biological Conservation, 32: 299-307.

Cullis, J. D., Gillis, C. A., Bothwell, M. L., Kilroy, C., Packman, A. \& Hassan, M. (2012). A conceptual model for the blooming behavior and persistence of the benthic mat-forming diatom Didymosphenia geminata in oligotrophic streams. Journal of Geophysical Research, 117, G00N03, doi:10.1029/2011JG001891.117(G2).

Díaz, M., Pedrozo, F., Reynolds, C. \& Temporetti, P. (2007). Chemical composition and the nitrogenregulated trophic state of Patagonian lakes. Limnologica, 37: 17-27.

Fransozo, A., Costa, R. C., Reigada, A. L. D. \& Nakagaki, J. M. (2003). Population structure of Aegla castro Schmitt, 1942 (Crustacea: Anomura: Aeglidae) from Itatinga (SP), Brazil. Acta Limnologica Brasiliensia, 15: 13-20.

Gillis, C.-A. \& Chalifour, M. (2010). Changes in the macrobenthic community structure following the introduction of the invasive algae Didymosphenia geminata in the Matapedia River (Quebec, Canada). Hydrobiologia, 647: 63-70.

Gordon, N. D., McMahon, T. A., Finlayson, B. L., Gippel, C. J. \& Nathan, R. J. (2004). Stream hydrology: an introduction for ecologists. Chichester: John Wiley and Sons.

Jara, C. G., Rudolph, E. H. y González, E. R. (2006). Estado del conocimiento de los malacostraceos dulceacuícolas de Chile. Gayana, 70 (1): 40-49.

Jellyman, P. G., \& Harding, J. S. (2016). Disentangling the stream community impacts of Didymosphenia geminata: How are higher trophic levels affected? Biological Invasions, 18(12): 3419-3435.

Kara, H. \& Sahin, B. (2001). Epipelic and epilithic algae of Degirmendere River (Trabzon - Turkey). Turkish Journal of Botany, 25: 177-186.

Kawecka, B. \& Sanecki, J. (2003). Didymosphenia geminata in running waters of southern Poland - symptoms of change in water quality? Hydrobiologia, 495: 193-201.

Kilroy, C. (2004). A new alien diatom, Didymosphenia geminata (Lyngbye) Schmidt: its biology, distribution, effects and potential risks for New Zealand fresh waters. NIWA, Client Report: CHC2004-128. Christchurch, New Zealand.

Kilroy, C., Snelder, T. H., Floerl, O., Vieglais, C. \& Dey, K. L. (2008). A rapid technique for assessing the suitability of areas for invasive species applied to New Zealand's rivers. Diversity and Distributions, 14: 262-272.

Kilroy, C., Larned, S. T. \& Biggs, B. J. F. (2009). The non-indigenous diatom Didymosphenia geminata alters benthic communities in New Zealand rivers. Freshwater Biology, 54: 19902002.

Kilroy, C. \& Bothwell, M. (2012). Didymosphenia geminata growth rates and bloom formation 
Macchi, P. J., Cussac, V. E., Alonso, M. F. \& Denegri, M. A. (1999). Predation relationships between introduced salmonids and the native fish fauna in lakes and reservoirs in northern Patagonia. Ecology of Freshwater Fish, 8: 227-236.

Medina-Vogel, G. \& González-Lagos, M. A. (2008). Habitat use and diet of endangered southern river otter Lontra provocax in a predominantly palustrine wetland in Chile. Wildlife Biology, 14: 211-220.

Montecino, V., Molina, X., Kumar, S., Castillo, M. L. \& Bustamante, R. O. (2014). Niche dynamics and potential geographic distribution of Didymosphenia geminata (Lyngbye) M. Schmidt, an invasive freshwater diatom in Southern Chile. Aquatic Invasions, 9(4): 507-519.

Morrone, J. J. \& Lopretto, E. C. (1994). Distributional patterns of Freshwater Decapoda (Crustacea: Malacostraca) in South America: a panbiogeographican approach. Journal of Biogeography, 21: 97-109.

Noga, T. (2003). Dispersion of Didymosphaenia geminata in the flowing waters of southern Poland - new sites of species occurrence in the Orawska watershed and the Orawska Basin. Oceanological and Hydrobiological Studies, 32: 159-170.

Parra, C. A., Barria, E. M. \& Jara, C. G. (2011). Behavioural variation and competitive status in three taxa of Aegla (Decapoda: Anomura: Aeglidae) from two-community settings in Southern Chile. New Zealand Journal of Marine and Freshwater Research, 45: 249-262.

Quinn, G. \& Keough, M. (2002). Experimental design and data analysis for biologist. UK. Cambridge University Press.

Reid, B. \& Torres, R. (2014). Didymosphenia geminata invasion in South America: ecosystem impacts and potential biogeochemical state change in Patagonian rivers. Acta Oecologica, 54: 101-109.

Rudolph, E. H. (2002). Sobre la biología del Camarón de río Samastacus spinifrons (Philippi 1882) (Decapoda, Parastacidae). Gayana, 66 (2): 147-159.

Sastre, A. V., Santinelli, N. H., Bauer, G. A., Ayesterán, M. G. \& Uyua, N. M. (2013). First record of the invasive diatom Didymosphenia geminata (Lyngbye) Schmidt in a Patagonian Andean river of Argentina. Biolnvasions Records, 2: 11-17.

Sepúlveda, M. A., Valenzuela, A. E. J., Pozzi, C., Medina-Vogel, G. \& Chehébar, C. (2015). Lontra provocax. The IUCN Red List of Threatened Species 2015: e.T12305A21938042. http:// dx.doi.org/10.2305/IUCN.UK.2015-2.RLTS. T12305A21938042.en.

Spaulding, S. A. \& Elwell, L. (2007). Increase in nuisance blooms and geographic expansion of the freshwater diatom Didymosphenia geminata. U.S. Geological Survey Open-File Report $2007-$ 1425.

Stevenson, R. J., Hill, B. H., Herlihy, A. T., Yuan, L. L. \& Norton, S. B. (2008). Algal-P relationships, thresholds, and frequency distributions guide nutrient criterion development. Journal of the North American Benthological Society, 27: 783-799.

Sundareshwar, P. V., Upadhayay, S., Abessa, M., Honomichl, S., Berdanier, B., Spaulding, S. A. \& Trennepohl, A. (2011). Didymosphenia geminata: Algal blooms in oligotrophic streams and rivers. Geophysical Research Letters, 38(10): L10405. doi: 10.1029/2010GL046599.

Taylor B. W. \& Bothwell, M. L. (2014). The origin of invasive microorganisms matters for science, policy, and management: the case of Didymosphenia geminata. BioScience, 64 (6): 531-538. 